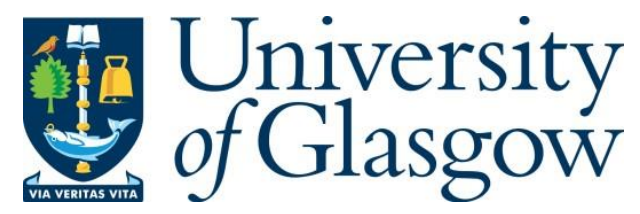

Yang, F., Le Kernec, J., Fioranelli, F. and Liu, Q. (2021) Shape Feature Aided Target Detection Method for Micro-drone Surveillance Radar. In: IET International Radar Conference 2020, Chongqing City, China, 4-6 Nov 2020, pp. 390-395. ISBN 9781839535406 .

There may be differences between this version and the published version. You are advised to consult the publisher's version if you wish to cite from it.

http://eprints.gla.ac.uk/245578/

Deposited on: 6 July 2021

Enlighten - Research publications by members of the University of Glasgow http://eprints.gla.ac.uk 


\title{
SHAPE FEATURE AIDED TARGET DETECTION METHOD IN MICRO-DRONE SURVEILLANCE RADAR
}

\author{
Fawei Yang ${ }^{1}$, Julien Le Kernec ${ }^{2}$, Francesco Fioranelli', Quanhua Liu ${ }^{1 *}$ \\ ${ }^{1}$ Key Laboratory of Electronic and Information Technology in Satellite Navigation (Beijing Institute of \\ Technology), Ministry of Education, Beijing, China \\ ${ }^{2}$ James Watt School of Engineering, University of Glasgow, Glasgow, United Kingdom \\ ${ }^{3}$ Department of Microelectronics, TU Delft, Delft, The Netherlands \\ *liuquanhua@bit.edu.cn
}

Keywords: FEATURE AIDED, MICRO-DRONE DETECTION, SHAPE FEATURE EXTRACTION

\begin{abstract}
This paper presents a shape feature aided target detection method in micro-drone surveillance radar in order to mitigate the false alarms caused by the ground clutter. The method consists of segmentation threshold selection method based on target measurements and shape feature extraction method based on Hu moments. Then the performance of the proposed method is verified by the real radar system. Field experiment using DJI phantom 3 is conducted and the measured data is analysed. The results shows that although there exist some limitations, the proposed method has good performance on eliminating the false alarms caused by the strong ground clutter in micro-drone detection and improving the target tracking accuracy.
\end{abstract}

\section{Introduction}

In recent years, with the development of technology, the production cost of the micro-drones, also called unmanned aerial vehicles (UAVs), has been greatly reduced, and commercial micro-drones have enjoyed explosive growth on the global market [1]. Micro-drones are in small size, light weight, easy to carry, and are widely used in daily entertainment such as travelling and filming. At the same time, industrial drones play an irreplaceable role in agriculture, industry, special operations and military use [2]. According to an investigation reported by Tsinghua University in 2018, the global UAV market will reach 3.3 million UAV shipments in 2019, of which 344 thousand UAVs will be commercial UAVs. Micro-drones are increasingly entering people's lives.

However, the rapid development of micro-drones has also brought many challenges to the global security. One of the most common incidents is unauthorized drone flights interfering with the normal take-off and landing of civil aviation aircraft at the airport [3]. In the latest years, airports around the world have reported more and more UAV interference incidents, which have greatly affected the order of the airports and also the individual safety of the passengers. In addition, the destruction of the personal privacy by microdrones is also a potential risk. Furthermore, micro-drones can be easily used by terrorists to carry out attacks [4]. Therefore, it is extremely necessary to develop efficient micro-drones surveillance and detection system.

People have developed a variety of detection systems, such as sonar, video, infrared, and radar to detect micro-drones. Among all the detection methods, the radar has the unique performance regardless of the weather and light conditions, and has greater advantages [5]. However, the detection of micro-drones is still a great challenge for radar. Micro-drones are mostly made of plastic, which results in extremely small radar cross section (RCS) [6, 7]. At the same time, the flying height of the micro-drones is very low, and the echo of them can be seriously affected by ground clutter during radar detection, resulting in large amount of false alarms.

Most of the radar systems for micro-drone detection in the literatures are based on frequency modulated continuous waveform (FMCW) radar [8-11], and there are few works on the system using pulse-Doppler (PD) radar. PD radar enjoys relatively high transmit power as well as long operating range compared to FMCW radar. In this paper, we proposed a shape feature aided target detection method for micro-drone surveillance radar using PD architecture in order to mitigate the false alarms caused by high scattering points on the ground. The proposed method composes of segmentation threshold selection method based on target measurements and shape feature extraction method based on $\mathrm{Hu}$ moments. The performance of the proposed method is verified by the PD radar system developed by the author's research group, showing good feasibility in mitigating the false alarms caused by the clutters in micro-drone detection.

The remaining of the paper is organized as follows. Section II describes the segmentation method and the shape feature extraction method. Section III presents the experimental results of the proposed method where the PD radar systems is introduced and the field measured data is processed to verify the feasibility and performance of the method. Finally, section IV concludes the paper and discusses the future analysis. 


\section{Shape Feature Extraction and Analysis}

\subsection{Segmentation Threshold Selection Method Based on Target Measurements}

The shape extraction is generally based on the segmentation of the target or corresponding area in the image. Image segmentation is a process of dividing an image into several regions with different properties. The most widely used method on image segmentation is based on threshold. The specific steps are as follows:

(1) Convert the input image to gray image.

(2) Calculate the segmentation threshold.

(3) Convert the gray image to binary image.

(4) Eliminate the connected domains that are smaller than the threshold in the binary image.

(5) Return the starting position and the row/column width of the connected domains that are higher than the threshold.

When it comes to the LSS target detection in complex electromagnetic environment, conventional image segmentation method such as OTSU face the limitation that the strong clutter makes the segmentation threshold too large for the weak target area to be stably segmented. Therefore, we propose a segmentation threshold selection method based on target measurements, which uses the CFAR detection results to determine the segmentation threshold. This method will ensure the gray value of the target is greater than the segmentation threshold, thereby stably segmenting the target areas. The flow chart of the algorithm is shown Figure 1:

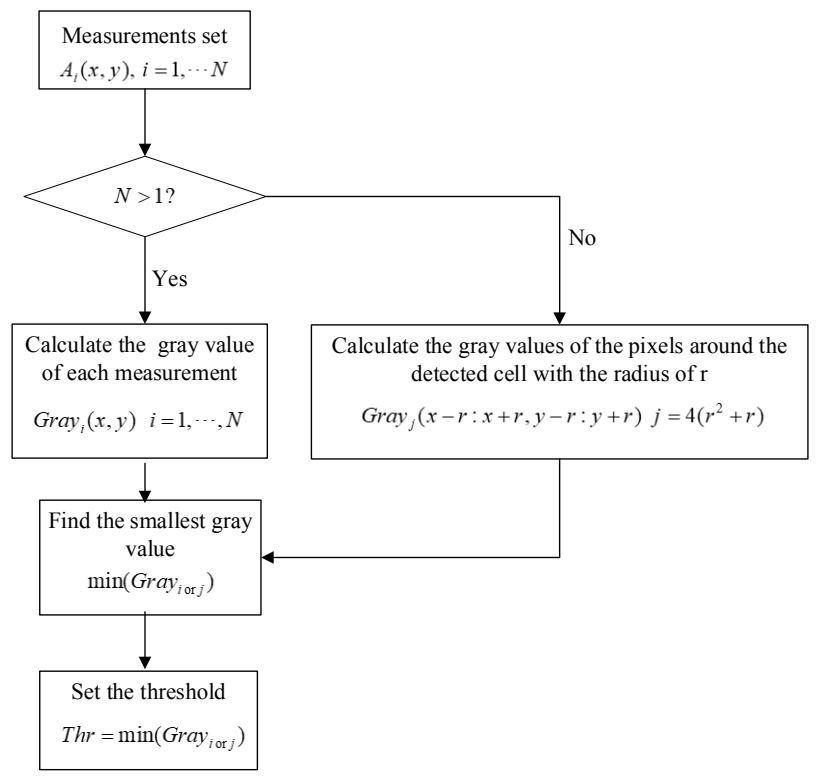

Figure 1 Flow chart of the segmentation threshold selection method based on target measurements

Specific steps are as follows:

(1) Extract the detection measurements after CFAR and clustering $A_{i}(x, y), i=1, \cdots N$, where $N$ represents the size of the measurements, $x$ and $y$ represent the index of range and Doppler cells on the range-Doppler map.

(2) If the size of the measurements is greater than 1, then calculate the corresponding gray value of each measurement, and set the smallest gray value as the segmentation threshold.

(3) If the size of the measurements is 1 , then calculate the gray values of the pixels around the detected cell with the radius of $r$, and set the smallest gray value as the segmentation threshold. The value of $r$ is determined according to the size of the detected target and the range resolution of the radar, which is shown in Figure 2

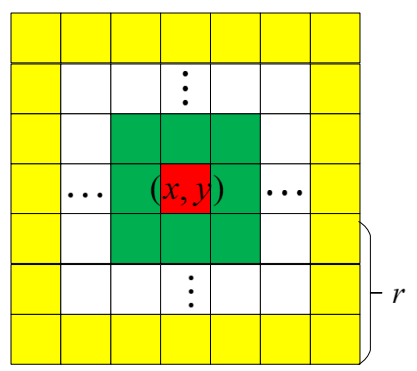

Figure 2 The radius for calculating the gray values of the pixels around the detected cell

\subsection{Shape Feature Extraction Method Based on Hu Moments}

When using segmented regions to extract shape features, we usually select the length, width, and invariant moments as metrics. The basic idea of the invariant moment method is to use the moments that are insensitive to image transformation to describe the shape feature. Here we use $\mathrm{Hu}$ moments to extract shape features. Assuming that the function of the digital image is $f(x, y)$, its $p+q$-th order geometric moment and central moment are defined as follows:

$$
\begin{gathered}
m_{p q}=\sum_{y=1}^{N} \sum_{x=1}^{M} x^{p} y^{q} f(x, y) \quad p, q=0,1,2 \ldots \\
\mu_{p q}=\sum_{y=1}^{N} \sum_{x=1}^{M}(x-\bar{x})^{p}(y-\bar{y})^{q} f(x, y) \quad p, q=0,1,2 \ldots
\end{gathered}
$$

where $\mathrm{M}$ and $\mathrm{N}$ represent the length and width of the segmented regions, $\bar{x}$ and $\bar{y}$ represent the image centre which are defined as:

$$
\begin{aligned}
& \bar{x}=m_{10} / m_{00} \\
& \bar{y}=m_{01} / m_{00}
\end{aligned}
$$

The normalized central moment is defined as:

$$
\eta_{p q}=\mu_{p q} / \mu_{00}^{\rho}
$$

where $\rho=\frac{p+q}{2}+1$. 
Using the normalized second and third order central moments, 7 invariant moments can be constructed as shape features:

$$
\begin{aligned}
\Phi 1= & \eta_{20}+\eta_{02} \\
\Phi 2= & \left(\eta_{20}-\eta_{02}\right)^{2}+4 \eta_{11}^{2} \\
\Phi 3= & \left(\eta_{30}-3 \eta_{12}\right)^{2}+\left(3 \eta_{21}-\eta_{03}\right)^{2} \\
\Phi 4= & \left(\eta_{30}+\eta_{12}\right)^{2}+\left(\eta_{21}+\eta_{03}\right)^{2} \\
\Phi 5= & \left(\eta_{30}-3 \eta_{12}\right)\left(\eta_{30}+\eta_{12}\right)\left(\left(\eta_{30}+\eta_{12}\right)^{2}-3\left(\eta_{21}+\eta_{03}\right)^{2}\right) \\
& +\left(3 \eta_{21}-\eta_{03}\right)\left(\eta_{21}+\eta_{03}\right)\left(3\left(\eta_{30}+\eta_{12}\right)^{2}-\left(\eta_{21}+\eta_{03}\right)^{2}\right) \\
\Phi 6= & \left(\eta_{20}-\eta_{02}\right)\left(\left(\eta_{30}+\eta_{12}\right)^{2}-\left(\eta_{21}+\eta_{03}\right)^{2}\right) \\
& +4 \eta_{11}\left(\eta_{30}+\eta_{12}\right)\left(\eta_{21}+\eta_{03}\right) \\
\Phi 7= & \left(3 \eta_{21}-\eta_{03}\right)\left(\eta_{30}+\eta_{12}\right)\left(\left(\eta_{30}+\eta_{12}\right)^{2}-3\left(\eta_{21}+\eta_{03}\right)^{2}\right) \\
& -\left(\eta_{30}-3 \eta_{12}\right)\left(\eta_{21}+\eta_{03}\right)\left(3\left(\eta_{30}+\eta_{12}\right)^{2}-\left(\eta_{21}+\eta_{03}\right)^{2}\right)
\end{aligned}
$$

Figure 3 The radar system

\begin{tabular}{ll}
\hline Parameter & Parameter value \\
\hline Frequency & S band \\
Instantaneous Bandwidth & $40 \mathrm{MHz}$ \\
Waveform Type & DDMA \\
Pulse repetition time (PRT) & $40 \mathrm{us}$ \\
Coherent Processing Interval (CPI) & $40 \mathrm{~ms}$ \\
Data rate & $0.25 \mathrm{~Hz}$ \\
\hline
\end{tabular}

The Hu moments analysis is performed on the extracted segmented regions, and the flow chart of the method is shown in Figure 3. The specific steps are as follows:

(1) Traverse all the segmented areas, and extract the starting position of each segmented area as well as the corresponding row and column width.

(2) Extract the gray value of each pixel in the segmentation area to form a Length $_{i} \times W_{i d t h}$ matrix $\operatorname{Gray}_{i}(x, y)$, where $x \in x_{i} \sim x_{i}+$ Length $_{i}-1, \quad y \in y_{i} \sim y_{i}+$ Width -1 .

(3) Calculate the zero and first order geometric moment, the image centre, and the normalized second and third order central moments of the segmented regions.

(4) Calculate the seven $\mathrm{Hu}$ moments of the image.

(5) Construct shape feature vector.

\section{Experimental Results and Verification}

\subsection{Measurement Configuration}

We choose the micro-drone surveillance radar designed and constructed by the author's research group as the platform to verify the feasibility of the proposed method. The radar is working at $\mathrm{S}$ band with the bandwidth of $40 \mathrm{MHz}$. For air surveillance use, the radar utilize pulse-Doppler processing with the pulse repetition time (PRT) of 40us and the coherent processing interval (CPI) of $40 \mathrm{~ms}$. The radar antenna keeps scanning the airspace in azimuth by mechanical rotating and achieve digital beam forming (DBF) in elevation by utilizing multi input and multi output (MIMO) technique. Also for the radar waveform, we choose Doppler division multiple access (DDMA) waveform to ensure orthogonal transmitting. The radar system is shown in Figure 3 and the main parameters of the system are shown in Table 1 [12-14].
Table 1 System parameters

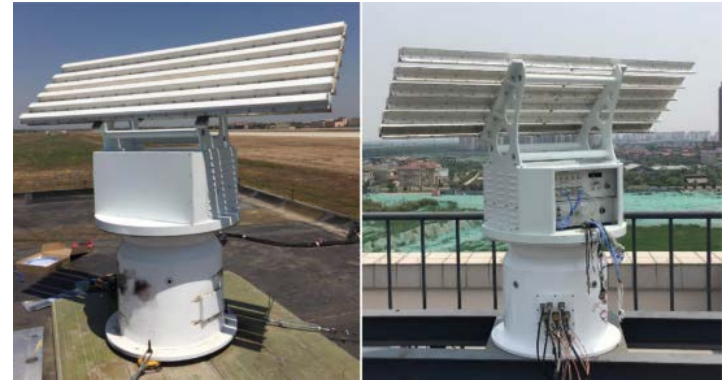

The micro-drone detection experiment is conducted in an airport. The detailed information of the scene is shown in Figure 4. The radar (yellow dot) is deployed on the roof of one observation room which is $3 \mathrm{~m}$ above the ground. We choose micro-Drone DJI Phantom 3 as the target and make it fly along the radial direction of the radar back and forth.

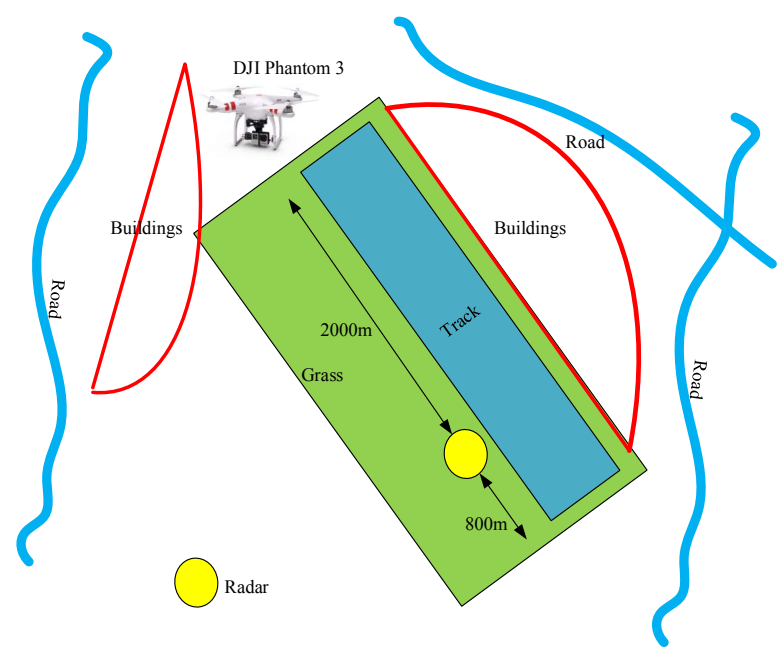

Figure 4 The experimental scene

\subsection{Shape Feature Extraction Results}

The range-Doppler (RD) map for one CPI after pulse compression and Doppler processing is shown in Figure 6 (a) from where the echo of the micro-drone is highlighted by black box. The segmentation result using OTSU is shown in Figure 5 (b). The threshold determined by OTSU method cannot segment the target area and display it in the binary image, indicating that the gray value of the target is less than the threshold. The OTSU method is invalid in this situation. 
The reason is that the gray value of the strong clutter makes the threshold too high for the weak target (micro-drone) to be segmented.

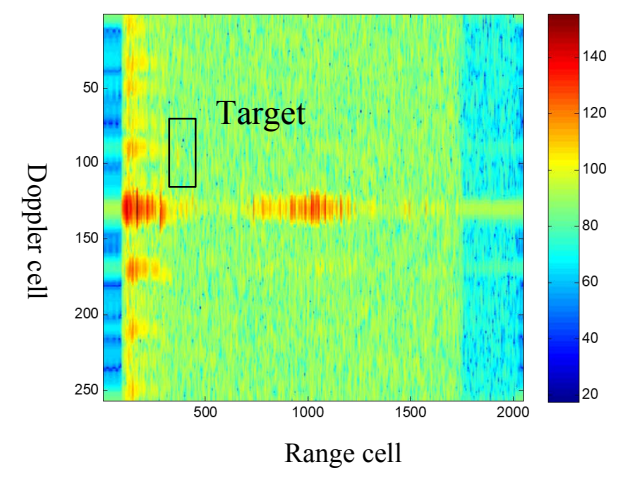

$a$

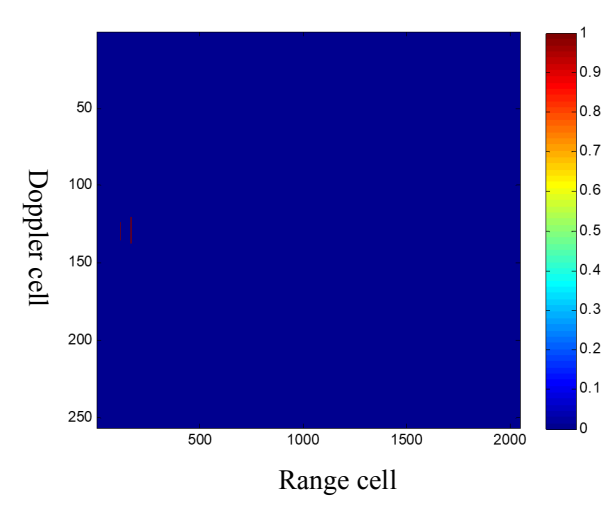

Figure 5

(a) Original RD map with target

(b) The binary image after segmentation using OTSU

The segmentation result using the proposed method is shown in Figure 7. The detection dots after CFAR and clustering for the same CPI is shown in Figure 6 (a), and there are four dots which belong to the drone and three clutters. The segmentation result using the proposed method is shown in Figure 7 (b), from which the target region and the clutter regions can all be segmented by the proposed method.

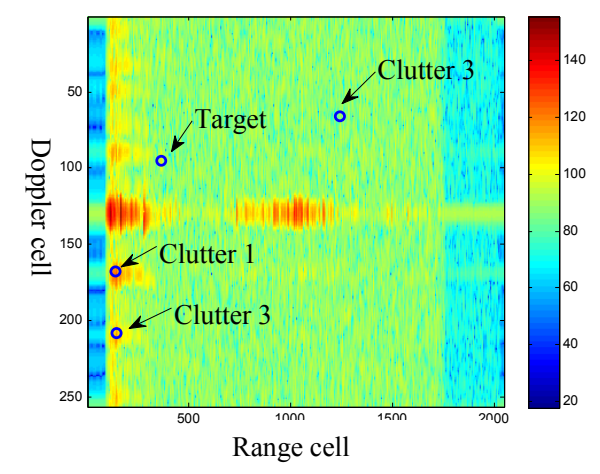

$a$

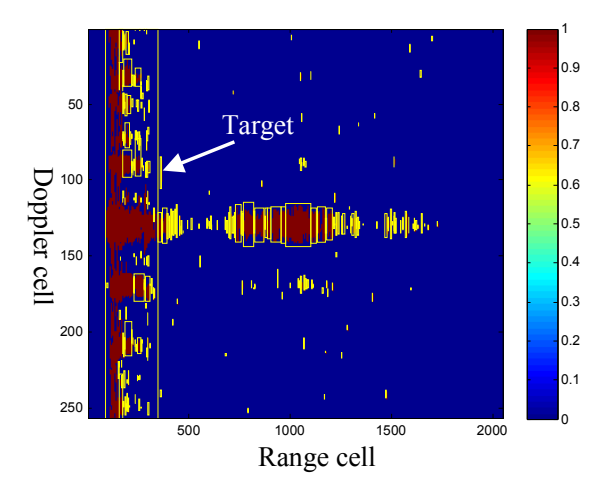

Figure 6
(a) The detection dots after CFAR and clustering
(b) The binary image after segmentation using the proposed method

Based on the segmented area of the target and clutter in Figure 7 (b), the Hu moments and the length and width of the smallest rectangle containing the segmented regions are calculated, and the results are shown in Table 2.

Table $2 \mathrm{Hu}$ moments of the segmented regions

\begin{tabular}{ccccc}
\hline & Clutter 1 & Clutter 2 & Clutter 3 & Target \\
\hline Length & 256 & 256 & 5 & 21 \\
Width & 254 & 254 & 2 & 6 \\
$\Phi 1$ & 0.2971 & 0.2971 & 0.2188 & 1.4305 \\
$\Phi 2$ & 0.0013 & 0.0013 & 0.0283 & 0.1754 \\
$\Phi 3$ & 0.0165 & 0.0165 & 0 & 0.0019 \\
$\Phi 4$ & 0 & 0 & 0 & 0 \\
$\Phi 5$ & 0 & 0 & 0 & 0 \\
$\Phi 6$ & 0 & 0 & 0 & 0 \\
$\Phi 7$ & 0 & 0 & 0 & 0 \\
\hline
\end{tabular}

It can be seen from Table 2 that the Hu moments $\Phi 1$ and $\Phi 2$ among the seven can effectively distinguish between the target and the clutter. In addition, the length and width of the smallest rectangle of the segmented region also have good discrimination of the target and the clutter. Hence the shape feature vector can be constructed as

$$
f(i)=\left[\text { Length }_{i}, \text { Width }_{i}, \Phi 1_{i}, \Phi 2_{i}\right]
$$

to assist target detection.

\subsection{Shape Feature Aided Detection Results on Micro-drone}

We employ the proposed method in processing the field measured data of the micro-drone to assist target discrimination. The DJI Phantom 3 is flying back and forth along the radial direction of the radar from $500 \mathrm{~m}$ to $1500 \mathrm{~m}$. Apply the conventional target detection method and the proposed shape feature aided detection method respectively, and the target detection and tracking results is shown in 
Figure 7, where the dots represents the detection measurements and the triangles represents the tracking results of the target.

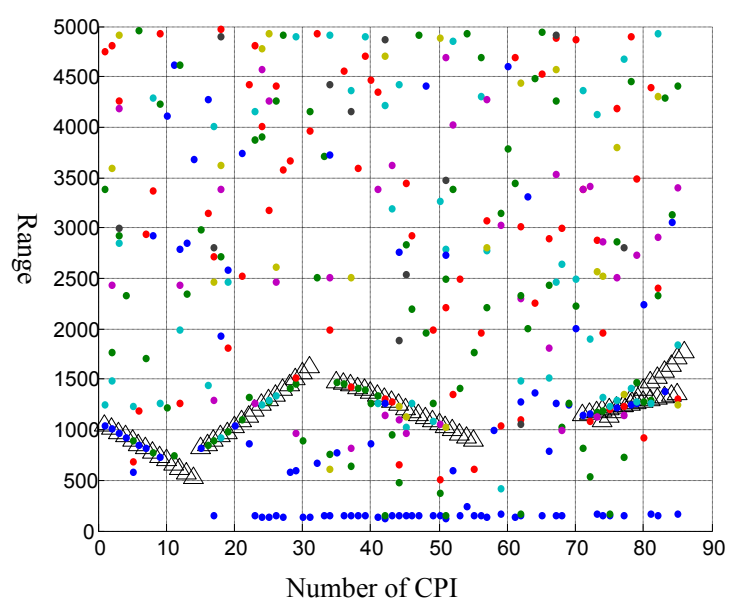

$a$

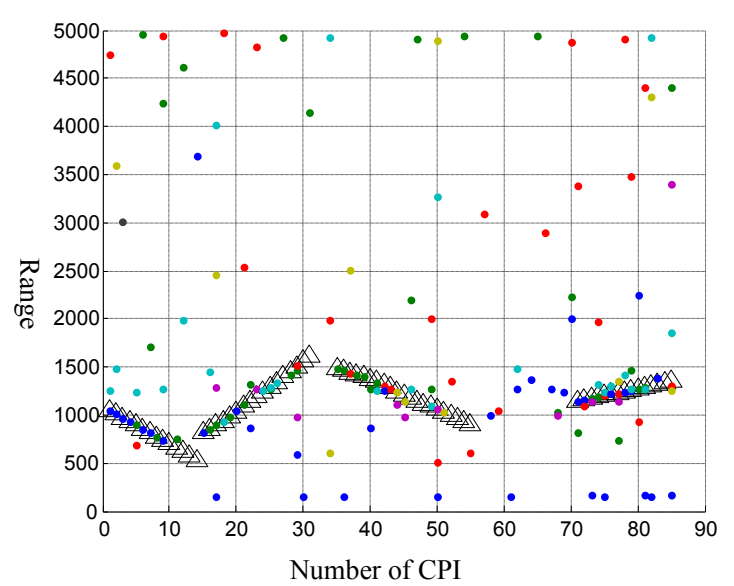

$b$

Figure 7

(a) The detection and tracking results of the micro-drone using conventional method

(b) The detection and tracking results of the micro-drone using proposed method

It can be seen from Figure 7 that the proposed shape feature aided detection method can effectively distinguish the target and the clutter and eliminate a large number of false alarms. Also the proposed method is helpful to correct some false tracks, improving the tracking accuracy.

\section{Conclusion}

In this paper, we presents a shape feature aided target detection method in micro-drone surveillance radar in order to mitigate the false alarms caused by the ground clutter. The method is described in detail and the performance of the method is verified using PD radar system developed by the author's research group. The results shows that although there exist some limitations, the proposed method has good performance on eliminating the false alarms caused by the strong ground clutter in micro-drone detection and improving the target tracking accuracy.

Future work includes the research on discriminating similar target such as the micro-drones with birds, cars and human.

\section{Acknowledgements}

This work was supported by the National Natural Science Foundation of China (Grant No. 61771050, 61671065 and 61490693 ) and the 111 Project of China under Grant B14010.

\section{References}

[1] Ritchie, M., Fioranelli, F., Griffiths, H.: 'Monostatic and bistatic radar measurements of birds and micro-drone', 2016 IEEE Radar Conference (RadarConf), Philadelphia, USA, 2016, pp. 1-5

[2] De Quevedo, Á. D., Urzaiz, F. I., Menoyo, J. G., López, A. A.: 'Drone Detection and RCS Measurements with Ubiquitous Radar', 2018 International Conference on Radar (RADAR), Brisbane, Australia, 2018, pp. 1-6

[3] Aldowesh, A., Alnuaim, T., \& Alzogaiby, A.: 'SlowMoving Micro-UAV detection with a small scale Digital Array Radar', 2019 IEEE Radar Conference (RadarConf), Boston, USA, 2019, pp. 1-5

[4] De Quevedo, Á. D., Urzaiz, F. I., Menoyo, J. G., \& López, A. A.: 'Drone detection and radar-cross-section measurements by RAD-DAR', IET Radar, Sonar \& Navigation, 2019, 13, (6), pp. 1437-1447

[5] Jahangir, M., \& Baker, C. J.: 'L-band staring radar performance against micro-drones', 2018 19th International Radar Symposium (IRS), Bonn, Germany, 2018, pp. 1-10

[6] Patel, J. S., Fioranelli, F., \& Anderson, D.: 'Review of radar classification and RCS characterisation techniques for small UAVs or drones', IET Radar, Sonar \& Navigation, 2018, 12, (9), pp. 911-919

[7] Rahman, S., \& Robertson, D. A.: 'In-flight RCS measurements of drones and birds at K-band and W-band', IET Radar, Sonar \& Navigation, 2019, 13, (2), pp. 300-309

[8] Ritchie, M., Fioranelli, F., Borrion, H., \& Griffiths, H.: 'Multistatic micro-Doppler radar feature extraction for classification of unloaded/loaded micro-drones', IET Radar, Sonar \& Navigation, 2017, 11, (1), pp. 116-124

[9] Zhang, W., Li, G., \& Baker, C.: 'Radar recognition of multiple micro-drones based on their micro-Doppler signatures via dictionary learning', IET Radar, Sonar \& Navigation, 2020, 14, (9), pp. 1310-1318

[10] Rahman, S., \& Robertson, D. A.: 'Classification of drones and birds using convolutional neural networks applied to radar micro-Doppler spectrogram images', IET Radar, Sonar \& Navigation, 2020, 14, (5), pp. 653-661

[11] Kim, B. K., Kang, H. S., \& Park, S. O.: 'Drone Classification Using Convolutional Neural Networks With 
Merged Doppler Images', IEEE Geoscience and Remote Sensing Letters, 2017, 14, (1), pp. 38-42

[12] Yang, F., Xu, F., Yang, X., \& Liu, Q.: 'DDMA MIMO radar system for low, slow, and small target detection', The Journal of Engineering, 2019, 2019, (19), pp. 5932-5935

[13] Hao, M., Yang, F., \& Liu, Q.: 'Slow-Time MIMO Radar Waveform Generator with Experimental Results', 2019 IEEE
International Conference on Signal, Information and Data Processing (ICSIDP), Chongqing, China, 2019, pp. 1-5

[14] Yang, F., Qu, K., Hao, M., Liu, Q., Chen, X., \& Xu, F.: 'Practical Investigation of a MIMO Radar System for Small Drones Detection', 2019 International Radar Conference (RADAR), TOULON, France, 2019, pp. 1-5 